\title{
Taxinomie et embryologie: une connexion ancienne et fructueuse
}

\author{
Herman Denis, Alain Collenot
}

Pour classer les grands groupes zoologiques, on utilise un système reposant sur le nombre et sur l'organisation des feuillets cellulaires qu'édifient les embryons au début de leur développement. Ce système taxinomique date de près d'un siècle. Il n'a pas été remis en cause malgré limportance croissante prise par la phylogenèse en matière de classification. On ne saurait s'en étonner. Il existe une correspondance entre développement et évolution. Si l'on compare des animaux apparentés, on apersoit entre les formes embryonnaires davantage de similitudes qu'entre les formes adultes. Plus les adultes se ressemblent, plus leur embryogenèse tend à diverger tardivement. Plus les adultes different, plus leur embryogenèse tend à diverger précocement. L'évolution montre une tendance analogue. Deux groupes proches proviennent d'une dichotomie tardive à partir d'une souche ancestrale. Des groupes éloignés ont divergé à une époque plus ancienne. En rapprochant les animaux suivant la similitude de leur développement, on retrace en quelque sorte leur évolution. On comprend dès lors qu'une classification cohérente ait émergé de deux sortes d'étude: celle qui consiste à comparer les modes de développement, et celle qui vise à reconstituer la phylogénie.

$\mathrm{m} / \mathrm{s} n^{\circ} 12$, vol. 11 , décembre 95

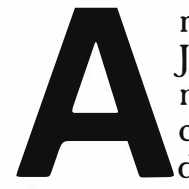
ristote $\left(I^{\mathrm{e}}\right.$ siècle avant J.-C.) nous a laissé la première trace écrite d'une classification raisonnée des animaux [1]. Il distingue deux catégories principales d'animaux: ceux qui sont pourvus de sang $(\varepsilon v \alpha \iota \mu \alpha)$ et ceux qui n'en possèdent pas $(\alpha v \alpha \mu \mu \alpha)$. En gros, les animaux sanguins correspondent aux vertébrés, tandis que les exsangues correspondent aux invertébrés. Dans un autre écrit, Aristote fait une étude comparative du développement embryonnaire [2]. Il propose un classement hiérarchique, où les mammifères occupent la position la plus élevée: ils produisent les jeunes les plus achevés. Puis viennent les requins ovovivipares, les oiseaux et les reptiles, etc., formant au total cinq groupes classés suivant une échelle de "perfection" dans l'embryogenèse. Aristote est bien le père de la biologie comparée. En deux ouvrages, il fonde deux disciplines biologiques apparemment sans rapport: la science du classement et celle du développement. Ces deux disciplines allaient évoluer de manière parallèle, la seconde servant en quelque sorte de guide à la première.

\section{Les bases de la taxinomie}

On appelle taxinomie ou systématique la branche du savoir qui vise à classer les êtres vivants en groupes ou taxons*, en fonction de critères rationnels. C'est une entreprise de longue haleine, puisqu'on estime que la faune terrestre comporte plusieurs millions d'espèces différentes. Suivant une évaluation déjà ancienne [3], il faudra presque mille ans pour terminer l'inventaire du monde animal commencé par Linné [4]. C'est une prédiction optimiste. Elle suppose un simple maintien du rythme où progressait au début du siècle la description des espèces animales. Étant donné le discrédit qui frappe les travaux de systématique, on peut craindre que l'achèvement de l'inventaire ne soit reporté sine die.

Sur quelles bases asseoir une classification rationnelle des êtres vivants? Tous les systèmes taxinomiques s'efforcent d'opérer un regroupement en fonction des ressemblances entre les organismes. Le critère de similitude impose la nécessité d'un classement hiérarchique, qui exclut toute connotation qualitative se référant à une prétendue perfection dans l'échelle des êtres. C'est Linné qui formalisa et imposa ce système [4]. Il

\footnotetext{
* Pour certains auteurs, le mot groupe a une signification plus large que le mot taxon. A leurs yeux, un taxon est un groupe monophylétique, dont les représentants dérivent par un même ancêtre d'une lignée préexistante d'êtres vivants.
} 
choisit de nommer les animaux et les plantes par deux noms latins, le premier désignant le genre et le second l'espèce. Dans la hiérarchie linnéenne, les espèces sont réunies en groupes de plus en plus vastes: genre, famille, ordre, classe, etc.

Les systèmes taxinomiques les plus anciens privilégiaient l'anatomie comme critère de similitude. Sur cette base, Lamarck fait une nette distinction entre vertébrés et animaux sans vertèbres [5]. Il ne fait que reprendre le classement d'Aristote. Cuvier distingue quatre embranchements: vertébrés, mollusques, articulés et rayonnés ou zoophytes [6]. Les articulés regroupent des animaux très différents, que l'on rangea par la suite dans des taxons distincts: les annélides et les arthropodes. Les zoophytes sont un fourre-tout, incluant entre autres les cnidaires, les plathelminthes, les nématodes et les échinodermes.

Le système de Cuvier va peu à peu se perfectionner au cours du XIX ${ }^{\mathrm{e}}$ siècle. Le terme d'embranchement subsistera, bien qu'on tende de nos jours à lui substituer celui de phylum. Par ces mots, on entend un groupe d'animaux construits suivant un plan d'organisation (Bauplan) particulier, différent de tous les autres $[3,7]$.

Depuis Cuvier, une tendance à long terme se manifeste dans les taxinomies successives que propose la littérature zoologique: le nombre de phylums s'accroît régulièrement. A l'heure actuelle, les spécialistes rangent les animaux multicellulaires (métazoaires) en une trentaine de phylums $[8,9]$. L'acception même du terme de phylum est assez fluctuante. C'est ainsi que, pour certains auteurs, les vertébrés forment un phylum indépendant $[10,11]$. Pour d'autres, ils font partie d'un ensemble plus vaste: les chordés $[3,9]$.

\section{Taxinomie et embryogenèse}

Il est facile de réunir en taxons des animaux qui présentent de grandes similitudes, comme un chat et une souris, un moineau et un pigeon. Les information suffisante. Mais si l'on a affaire à des animaux très différents, ces critères deviennent inopérants. Dès la première moitié du XIX ${ }^{\mathrm{e}}$ siècle, les biologistes tentèrent de surmonter la difficulté en comparant les formes embryonnaires des divers animaux. C'est von Baer qui les y engagea en formulant ses fameuses lois embryologiques [12]. La première de ces lois stipule que le développement fait apparaître les caractères généraux d'un grand groupe zoologique avant les caractères plus spécialisés. La deuxième loi constate que les caractères les moins généraux se développent à partir des caractères les plus généraux, jusqu'à ce que les caractères les plus spécialisés émergent.

Ces remarques frappent par leur pertinence. Toutefois, il faut les nuancer quelque peu. Comme von Baer luimême l'avait noté, les similitudes entre groupes voisins ne se manifestent pas toujours dès le début du développement. Prenons comme exemple les vertébrés. Les oufs de ces animaux ont des tailles très inégales. Ceux des oiseaux sont énormes. Ceux des mammifères sont petits et édifient rapidement une structure particulière (le trophoblaste), qui leur permet de se nourrir aux dépens de l'organisme maternel. Il s'ensuit que l'embryogenèse précoce (segmentation, gastrulation) se déroule suivant des modalités très différentes, suivant que la charge de l'œuf en vitellus est forte (sélaciens, reptiles, oiseaux), modérée (amphibiens) ou nulle (mammifères). Mais les embryons de tous les vertébrés deviennent plus semblables à mesure qu'ils se développent. Ils convergent en quelque sorte vers un goulet d'étranglement, qui définit le stade phylotypique, c'est-à-dire caractéristique du phylum. Pour les vertébrés, il s'agit du stade pharyngula [13-15]. Ensuite, les formes embryonnaires divergent de nouveau. La convergence évoquée a probablement une explication génétique. La morphogenèse post-gastruléenne est régie par un ensemble de gènes, dits homéotiques, dont le rôle consiste à modeler l'embryon suivant son axe primaire (oralaboral ou antéro-postérieur) [14, 15]. Ces gènes ont une structure et un mode d'action qui varient assez peu chez les divers représentants d'un même phylum [16].

Cette réserve faite, les lois de von Baer restent valables. Une conclusion s'impose: pour vérifier s'il convient de réunir en taxons des animaux sans parenté évidente, il faut étudier leur embryogenèse. C'est ce que firent les biologistes à partir de 1850. L'embryologie comparée prit son essor.

\section{Esquisse d'une classification}

Von Baer et ses contemporains avaient commencé par décrire le développement du poulet $[12,17,18]$. Ils s'étaient aperçus que l'embryon dispose ses cellules en trois feuillets superposés. On remarqua bientôt que les embryons des animaux les plus simples ne forment que deux feuillets [19]. S'appuyant sur ces observations, Ray Lankester proposa une classification des métazoaires en deux groupes majeurs: les didermiques ou diploblastiques, dont l'embryon comporte deux feuillets, et les tridermiques ou triploblastiques, dont l'embryon en édifie trois [20, 21]. Les didermiques regroupent trois phylums importants: les éponges, les cnidaires et les cténaires. Les tridermiques comprennent tous les autres phylums (figure 1).

Comment classer les animaux tridermiques? L'embryologie comparée suggéra quelques rapprochements inattendus. Kowalevsky découvrit l'existence d'affinités entre les vertébrés et les urochordés (aussi appelés prochordés ou tuniciers) [22]. Il remarqua la similitude que présente un têtard d'amphibien avec une larve d'ascidie. L'un et l'autre possèdent un tube nerveux dorsal ainsi qu'un élément de soutien, ébauche de colonne vertébrale: la notochorde. En étudiant leur développement, on s'aperçut que les vertébrés sont aussi apparentés aux échinodermes [23]. Les embryons des deux groupes exécutent de façon similaire les mouvements gastruléens, qui placent à l'intérieur de l'embryon les cellules du mésoderme et de l'endoderme. Ces déplacements cellulaires créent une ouverture dans la paroi de la blastula: le blastopore. Dans les deux groupes 


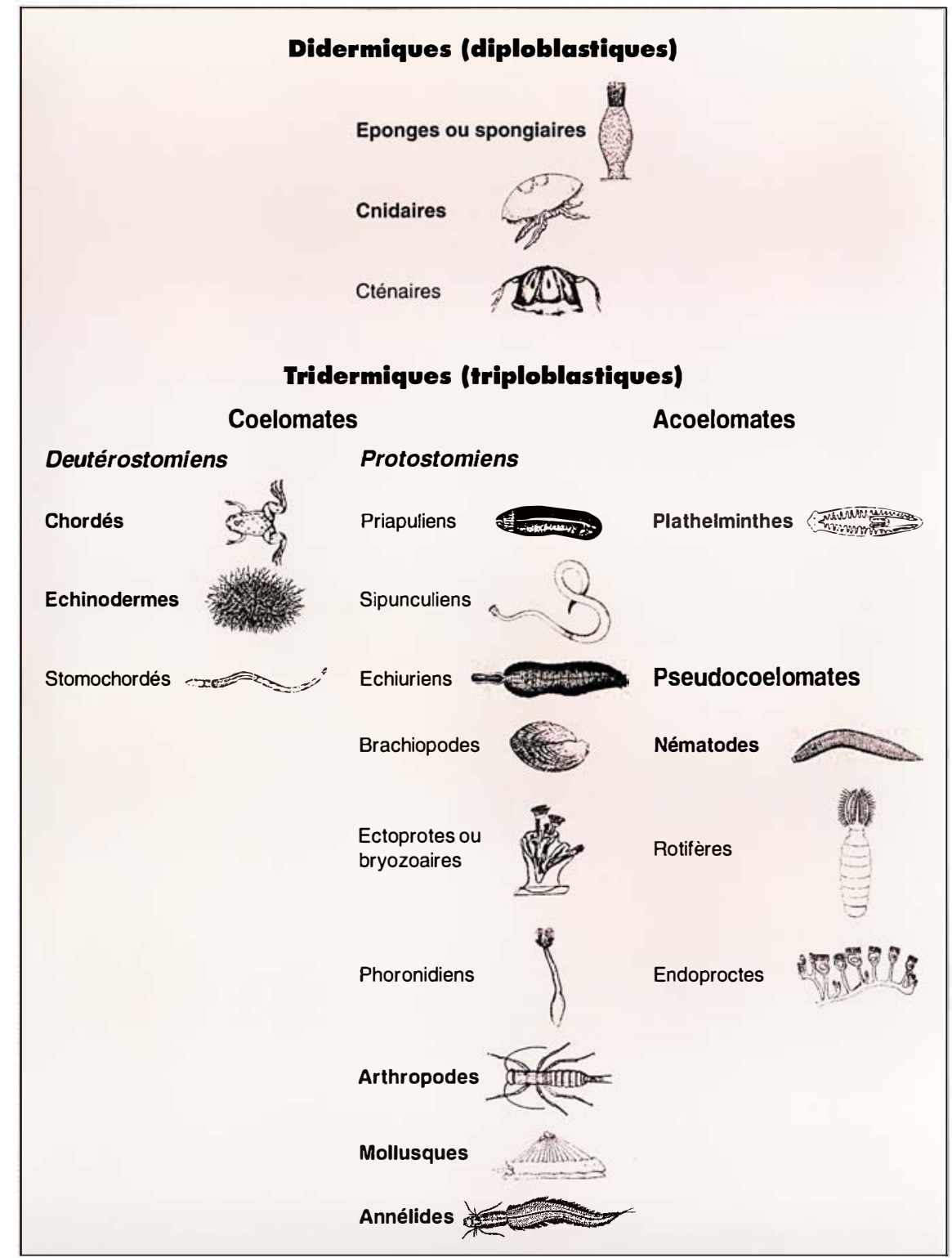

Figure 1. Bases embryologiques de la classification. Les métazoaires se répartissent en une trentaine de phylums, dont 19 sont ici figurés. On a indiqué en caractères gras le nom des phylums qui l'emportent sur les autres par l'abondance et la diversité de leurs représentants. Les métazoaires se subdivisent en didermiques et tridermiques suivant que leur embryon comporte deux ou trois feuillets. Les tridermiques se rangent en trois grandes catégories: les cœlomates, les acœlomates et les pseudocœlomates. Les cœlomates regroupent la majorité des phylums. Leur caractéristique commune est $d^{\prime}$ avoir un mésoderme organisé en vésicules closes. Ils se répartissent en deutérostomiens et en protostomiens, selon plusieurs critères embryologiques. Les acœlomates et les pseudocœlomates ont un mésoderme dépourvu de cavités. (D’après [9], modifié.) évoqués, le blastopore devient l'anus ou marque l'emplacement de cet orifice. La bouche s'ouvre plus tardivement.

Ces découvertes avaient de quoi surprendre. En effet, qu'y a-t-il de commun entre une grenouille, une ascidie - sorte d'outre à double ouverture, fixée au fond des mers et un oursin? A première vue, rien. Pourtant, tous ces animaux appartiennent à des groupes voisins, dont le développement précoce se déroule suivant des modalités comparables.

Ainsi se construisit peu à peu une classification fondée sur des critères embryologiques $[3,23]$. Les animaux tridermiques se classent en deux grandes catégories suivant la manière dont se structure le mésoderme au cours du développement embryonnaire (figure 1). Le mésoderme des cœlomates se creuse en une série de cavités, dont l'ensemble porte le nom de cœlome. Le mésoderme des acœlomates ne forme pas de cavités. On fait d'habitude une distinction entre les acœlomates proprement dits et les pseudocœlomates [3, 23]. Chez les premiers, le mésoderme forme un parenchyme compact qui remplit l'espace compris entre le tube digestif et la paroi du corps. Chez les seconds, une cavité occupe ce même espace. Il s'agit d'un pseudocœlome, assimilable à la cavité primaire de l'embryon (le blastocœle). Cette cavité n'est pas bordée par du mésoderme.

C'est encore en fonction de critères embryologiques que l'on divise les cœlomates en deutérostomiens et en protostomiens (figure 1). La distinction repose essentiellement sur la manière dont se déroule la gastrulation [3, 23]. Chez les deutérostomiens, l'anus provient du blastopore. Chez les protostomiens, le blastopore ne donne pas directement naissance à l'anus. Il coïncide en général avec la bouche [3, 23]. Il y a d'autres différences entre les deutérostomiens et les protostomiens [11, 14]. L'œuf des deutérostomiens se segmente de façon radiaire : les divisions se font dans un plan perpendiculaire ou parallèle à l'équateur. Chez certains pro- 
tostomiens, l'œuf se segmente suivant le mode spiral : à partir de la troisième division, les plans de clivage sont inclinés par rapport à l'équateur. Chaque blastomère d'un rang donné se décale de manière à chevaucher deux blastomères du rang inférieur. Au lieu de s'empiler les unes au-dessus des autres, les cellules se disposent suivant une spirale imaginaire qui apparaît quand on observe l'embryon par le dessus, c'est-à-dire par le pôle animal.

\section{Taxinomie et phylogenèse}

Près d'un siècle s'est écoulé depuis l'introduction du système taxinomique reposant sur l'étude de l'embryogenèse [23]. Pendant ce laps de temps, beaucoup de choses ont changé en matière de taxinomie. Les idées ont évolué tout autant que les méthodes. La phylogenèse a pris une importance croissante dans les recherches taxinomiques. Quel a été l'impact de ces changements sur le système séculaire de classification zoologique?

Pendant longtemps, la théorie de l'évolution a exercé peu d'influence sur la taxinomie. Pourtant, Darwin pensait que toute classification naturelle devait s'appuyer sur la généalogie [24]. Ce point de vue paraît justifié. Les êtres vivants diffèrent parce que leurs générations successives se sont progressivement modifiées. Chez les animaux, la reproduction sexuée favorise la descendance avec modification, car elle entretient une grande diversité génétique dans un groupe d'individus interféconds. La sélection naturelle avantage les individus les mieux adaptés au milieu. C'est en quelque sorte le moteur du changement. Le moteur ne s'arrête jamais. Plus le temps passe, plus son effet se fait sentir. Il arrive un moment où les individus d'une espèce* donnée cessent d'être interféconds. Certains d'entre eux s'isolent génétiquement, fondant ainsi une nouvelle espèce. Beaucoup de biologistes pensent que cela se produit quand de petits groupes se détachent à la périphérie d'une popula- soit le mécanisme invoqué pour expliquer la spéciation, il est clair aux yeux des évolutionnistes que toute similitude entre espèces, reflétée par la classification, repose sur une communauté d'origine, que l'on peut représenter par une succession de dichotomies.

Les préceptes darwiniens incitent à utiliser la paléontologie pour étayer la classification. Il s'agit de confirmer les regroupements opérés sur la base de comparaisons entre les organismes actuels. Malheureusement, la paléontologie ne permet pas de résoudre tous les problèmes. Elle n'est guère utile pour préciser les relations entre phylums. Considérons par exemple deux groupes rapprochés en fonction de critères embryologiques: les échinodermes et les vertébrés. Un lien entre ces deux phylums pourrait être établi grâce à des fossiles datant du début de l'ère primaire: les mitrates et les cornutes [26]. Ces étranges créatures appartiendraient à un groupe ancestral hypothétique: celui des calcichordés, dont les échinodermes et les vertébrés dériveraient [26]. Mais l'hypothèse des calcichordés n'a pas rencontré un grand écho parmi les paléontologues. Il semble qu'en fait les cornutes et les mitrates doivent être rangés dans le phylum des échinodermes [28].

On peut facilement expliquer l'absence de fossiles utilisables pour établir des parentés entre phylums: il a fallu peu de temps pour que surgissent des animaux bâtis suivant des plans d'organisation différents [29, 30]. La faune d'Ediacara constitue le plus ancien gisement de métazoaires identifiables, enfouis dans les montagnes d'Australie depuis quelque 600 millions d'années [29, 30]. Les

* La notion d'espèce est très difficile à définir. Elle suscite des discussions sans fin entre spécialistes. Il en va de même pour le processus de spéciation. Sans vouloir prendre parti dans ces débats, nous entendons par espèce un ensemble d'individus interfé conds. Bien entendu, cette définition ne vaut que pour les organismes pratiquant la reproduction sexuée. cnidaires paraissent déjà représentés dans la faune d'Ediacara. Comme à l'heure actuelle, ils étaient probablement subdivisés en quatre classes, sans intermédiaires reconnaissables [30]. La faune de Burgess révèle une seconde explosion de vie animale [30, 31]. Cette explosion se produisit il y a environ 530 millions d'années, à l'aube de l'ère primaire (début du cambrien). La plupart des phylums existants apparurent à cette époque, en 5 à 10 millions d'années [32, 33]. Ce laps de temps paraît remarquablement bref si on le compare à l'énorme intervalle $(2,5$ à 3 milliards d'années) qui sépare l'émergence des premières cellules de celle des premiers métazoaires [31].

\section{Les écoles \\ de systématique}

Même si la paléontologie n'offre pas une réponse à toutes les questions que se posent les systématiciens, il ne faut pas pour autant abandonner tout recours à la phylogenèse pour étayer la taxinomie. Déplorant le divorce prolongé entre généalogie et taxinomie, plusieurs zoologistes entreprirent de repenser les principes de classification [34-36]. Ces auteurs et leurs disciples forment une école, dite de la systématique évolutionniste. Ils accordent une grande importance au grade, qui se définit comme le niveau général d'organisation, fruit d'une évolution plus ou moins avancée [8]. La classification se fonde sur plusieurs critères: comparatif, numérique et généalogique, dont l'incidence peut se résumer comme suit [37]. S'il existe un écart morphologique important entre organismes, il convient de les ranger dans des taxons distincts. On attribue à un groupe riche en espèces un rang plus élevé qu'à un groupe pauvre. Chaque groupe constitué doit satisfaire au critère minimal de monophylie: ne comporter que des descendants d'un même ancêtre, sans nécessairement les réunir tous.

Deux autres écoles de pensée se disputent les faveurs des taxinomistes. L'une refuse tout recours à la généa-

$\mathrm{m} / \mathrm{s} n^{\circ} 12$, vol. 11 , décembre 95 
logie pour établir la classification. Ses partisans préconisent une taxinomie numérique ou phénétique. Les phénéticiens rejettent l'utilité de considérations théoriques, en particulier évolutives, pour asseoir la taxinomie [38]. La classification se bâtit en déterminant le degré de similitude globale entre les espèces. Les ressemblances se jugent suivant des critères quantitatifs. On doit fonder les rapprochements entre taxons sur le plus possible de caractères, sans chercher à faire la distinction entre ceux qui sont ancestraux et ceux qui sont dérivés.

Une troisième école l'emporte sur les deux autres [37, 39]. Fondée en 1950 par Hennig [40], cette école prône une systématique phylogénétique, fondée uniquement sur la généalogie. Les disciples de Hennig sont connus sous le nom de cladistes. Leur système de classification repose sur le clade, qui est un concept de nature phylogénétique. Par définition, le clade est un taxon monophylétique, qui réunit tous les descendants d'une espèce ancestrale. Le critère de monophylie réside dans l'existence de synapomorphies, c'est-à-dire de caractères dérivés (apomorphes) partagés par tous les membres d'un groupe. Aucune valeur n'est attribuée à la présence de caractères restés primitifs (plésiomorphes), parce que ces caractères ne révèlent rien concernant la parenté des espèces comparées. Pour les cladistes, toute espèce est par définition monophylétique. Quand une espèce ancestrale se divise en deux espèces filles, elle cesse d'exister en tant que telle. L'essentiel de la construction cladistique consiste à rapprocher les groupes frères. Elle aboutit à l'édification d'un cladogramme, figure dichotomique censée exprimer les relations phylogénétiques entre les taxons.

Les idées des cladistes mirent du temps pour s'imposer: environ trois décennies [39]. Elles permettent de réaliser un meilleur accord entre la classification et la phylogénie, comme le souhaitait Darwin. Cette amélioration vient du fait que les cladistes prennent en compte la signification évolutive des caractères pour former les taxons. L'analyse cladistique a d'autres mérites. En particu$\mathrm{m} / \mathrm{s} n^{\circ} 12$, vol.11, décembre 95 lier, elle introduit de la rigueur dans les travaux de systématique, qui en manquaient quelque peu [41]. A tout prendre, le concept de clade est bien plus clair et précis, donc plus facile à utiliser, que celui de grade.

\section{Les dendrogrammes moléculaires}

Le début des années 1960 marque l'entrée de la biologie moléculaire dans les études taxinomiques [42]. L'idée était de recourir aux gènes ou à leurs produits (ARN et protéines) pour classer les organismes. Cette idée semble légitime car ce sont les gènes qui conditionnent tous les caractères phénotypiques utilisables pour l'établissement de la taxinomie. Pourquoi ne pas remonter aux sources?

On commença par comparer les séquences de protéines de même fonction (cytochrome $\mathrm{C}$, hémoglobine, etc.). Les progrès étaient lents parce qu'il fallait purifier chaque protéine, la couper en peptides, puis séparer ceux-ci avant de commencer le séquençage proprement dit. Les premiers résultats étaient encourageants. Il s'avérait que les similitudes entre protéines diminuent à mesure que les animaux comparés s'éloignent dans la classification. L'approche moléculaire était donc validée par la morphologie et même par la paléontologie. L'accord paraissait si bon que la notion d'horloge moléculaire s'imposa: chaque protéine évolue à une vitesse constante, en accumulant dans chaque lignée animale un nombre fixe de substitutions d'acides aminés par million d'années [43]. Cependant, il se pourrait que la marche de l'horloge ne soit pas toujours bien régulière [44]. Certains auteurs contestent même son existence [44].

Ces succès initiaux stimulèrent l'ambition des taxinomistes moléculaires. Il était tentant d'élargir les comparaisons aux grands groupes zoologiques, en utilisant des macromolécules ubiquistes, tels que les ARN ribosomiques $18 \mathrm{~S}$ et $28 \mathrm{~S}$, les facteurs de traduction (EF-1 $\alpha, E F-2)$, ou les protéines du cytosquelette (tubuline, actine). De nouvelles techniques donnèrent une nouvelle impulsion aux recherches: le clonage moléculaire de l'ADN, puis le séquençage rapide de l'ADN et de l'ARN. Ainsi se constitua rapidement une banque de données contenant les séquences de plusieurs milliers de macromolécules. On vit apparaître un nombre croissant de figures dichotomiques (dendrogrammes) fondées sur des comparaisons entre séquences d'un acide nucléique (ARN) ou d'une protéine [45-56]. Ces constructions décrivent les similitudes entre les gènes spécifiant la macromolécule étudiée, plutôt que les relations de parenté unissant les organismes d'où provient cette macromolécule.

\section{Construction des dendrogrammes moléculaires}

Les premiers dendrogrammes étaient édifiés de façon artisanale. On alignait les séquences, puis on s'efforçait de relier les molécules en fonction de leurs similitudes structurales. Rapidement, l'informatique fit irruption dans ce domaine [57]. A l'heure actuelle, les dendrogrammes sont établis par ordinateur sur la base de logiciels utilisant des algorithmes développés à partir des méthodes d'analyse phénétique et cladistique [58]. Nourries d'un jeu de séquences alignées, les machines construisent des graphiques reliant les séquences suivant leur degré de similitude. Pour les phénéticiens, ces constructions ont une valeur purement taxinomique. Ce sont des phénogrammes. Pour les cladistes, elles reflètent la généalogie. Ce sont des cladogrammes, que l'on peut assimiler aux arbres phylogénétiques inventés par les biologistes du XIX ${ }^{e}$ siècle [39].

Pour aboutir à des phylogénies crédibles, les analyses de séquence doivent répondre à plusieurs exigences. En premier lieu, il faut que les séquences soient alignées correctement. Malheureusement, il n'existe pas encore de méthode automatique pour produire des alignements exacts. Dans ce domaine, il subsiste une certaine part d'arbitraire: on cherche en tâtonnant à aligner le 


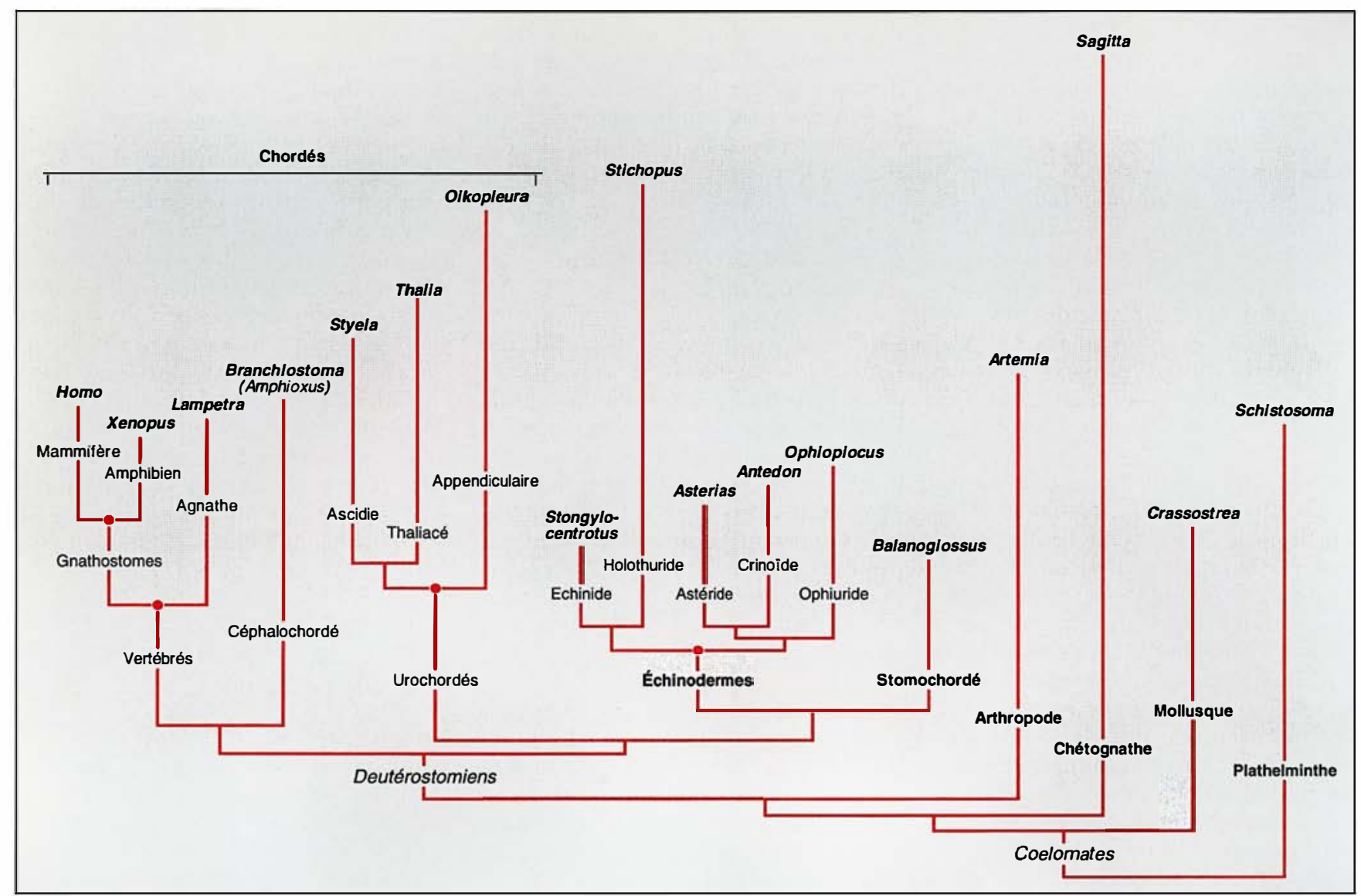

Figure 2. Un dendrogramme moléculaire. L'arbre a été édifié en comparant les séquences de 17 ARN ribosomiques 18S, appartenant à 17 genres (en caractères gras italiques), répartis en 7 phylums (en caractères courants gras). Les branches de l'arbre relient les molécules d'ARN en fonction des similitudes de leur séquence. Plus les molécules sont semblables, plus petite est la longueur cumulée des lignes verticales qui les unissent, en passant par tous les points de branchement (nœuds) intercalés. Le dendrogramme a été produit par un algorithme dit de distance, qui joint les séquences suivant le nombre de nucléotides identiques répertoriés dans les différentes molécules. Le test de bootstrap a permis d'évaluer la solidité de l'arbre. Il consiste à construire une grande quantité de dendrogrammes en omettant tour à tour une partie des nucléotides utilisés pour établir les distances entre les molécules. Retrait et remise se font au hasard. Les points rouges signalent les nœuds qui sont présents dans environ $90 \%$ des arbres produits, sur un total de 500. Suivant ce critère, les vertébrés forment un groupe monophylétique. II en va de même pour les urochordés et les échinodermes. On ne peut tirer aucune conclusion ferme concernant le statut des chordés (vertébrés + céphalochordés + urochordés), ni concernant celui des deutérostomiens. Ce dernier groupe paraît exclure le chétognathe Sagitta, ce qui contredit la position taxinomique que l'on attribue traditionnellement à cet animal [7, 10, 59]. Tel qu'il est présenté, l'arbre pourrait suggérer que les groupes ancestraux ont dans la classification un rang égal à celui qu'on accorde à leurs descendants actuels: phylum, classe, ordre, famille, etc. Ce serait une erreur: aucun taxon de rang supérieur à l'espèce ne saurait être considéré comme un ancêtre naturel [58]. (D'après [54], modifié.)

mieux possible les séquences, en les décalant si nécessaire pour compenser les résidus qui semblent absents ou en excès dans l'une ou l'autre molécule. En deuxième lieu, il faut que les arbres engendrés soient reproductibles quand le même jeu de sé- quences est soumis de manière répétée à l'analyse informatique. Pour évaluer la confiance que l'on peut avoir dans les arbres, on recourt souvent au test de cohérence interne connu sous le nom de bootstrap, qui consiste à échantillonner plusieurs fois les données et à vérifier qu'un même branchement apparaît dans une grande majorité de topologies engendrées $[52,58]$. En troisième lieu, il faut que des arbres compatibles (congruents) émergent de comparaisons portant sur des macro- 
molécules différentes. Parmi les analyses publiées [45-56], rares sont celles qui satisfont aux deux dernières exigences $[46,51]$. Les auteurs de ces travaux emploient le test de bootstrap pour éprouver la solidité des constructions qu'ils obtiennent. Afin d'en vérifier la congruence, ils utilisent les produits de différents gènes pour établir plusieurs séries d'arbres, qui sont ensuite comparées.

\section{Pérennité de la classification}

Les études moléculaires ne permettent pas toujours de tirer des conclusions définitives concernant les relations phylogénétiques entre les grands groupes de métazoaires [52, 55]. Prenons comme exemple une comparaison récente portant sur l'ARN ribosomique $18 \mathrm{~S}$. On constate que les vertébrés ont entre eux de plus fortes ressemblances qu'avec le reste des métazoaires (figure 2). C'est également vrai pour les urochordés et des échinodermes. On aurait donc affaire à des taxons monophylétiques. En revanche, on ne peut rien conclure concernant la monophylie des deutérostomiens. Au sein de ce groupe, les urochordés paraissent plus proches des échinodermes et des stomochordés que des vertébrés (figure 2). Mais à cet égard, la construction présentée n'est pas très solide: les valeurs de bootstrap obtenues sont trop faibles pour remettre en question les affinités que les recherches embryologiques et morphologiques ont établies entre les vertébrés et les urochordés [10, 22, 23, 59]. D'autres comparaisons tendent à établir de manière plus probante la monophylie des deutérostomiens [47], des cœlomates [45, 52, 55], des tridermiques et des didermiques [48, $50,52,55]$.

A ce jour, rares sont les phylums dont la position systématique ait été mise en cause par des phylogénies moléculaires d'une grande solidité. C'est peut-être le cas pour les némertiens, qu'il faudra sans doute rattacher aux cœlomates, plutôt qu'aux acœlomates [49, 56]. On $m / s n^{\circ} 12$, vol. 11, dérembre 95 devra sans doute aussi réviser la position des pogonophores et des chétognathes (figure 2): les comparaisons entre séquences d'ARN ribosomique $[53,54,56]$ n'ont pas confirmé les affinités souvent reconnues de ces groupes avec les deutérostomiens (chordés, échinodermes et stomochordés) [7, 10, 59]. Toutefois, il convient de considérer avec prudence les données expérimentales qui invitent à revoir la position des chétognathes (figure 2), parce que les gènes ribosomiques ont apparemment évolué plus vite dans la lignée qui conduit au chétognathe étudié (Sagitta) que dans celles qui aboutissent à beaucoup d'autres métazoaires. Cela risque de fausser le point d'insertion dans le dendrogramme du rameau menant à Sagitta [55].

On voit qu'en près de cent ans, peu de choses ont changé dans la classification des métazoaires [23]. Pour classer les grands groupes zoologiques, les auteurs contemporains adoptent en gros le système taxinomique fondé sur la manière dont se déroule le début du développement, et en particulier la gastrulation [7, 10, 59]. On ne peut qu'admirer la perspicacité des embryologistes du XIX ${ }^{e}$ siècle.

\section{Taxinomie et récapitulation}

Comment interpréter la pertinence de la classification zoologique reposant sur l'étude comparée du développement? Nous estimons que cette classification s'appuie sur une base conceptuelle saine, qui est la théorie des feuillets. Celle-ci se rattache à deux autres théories, de plus grande envergure: la théorie des récapitulations (ou loi biogénétique) et la théorie de l'évolution. Ces trois théories sont liées, puisque la théorie des feuillets est de nature récapitulative, et que la loi des récapitulations est elle-même de nature évolutionniste [60]. Pourtant, ces théories ont une influence très inégale sur la pensée scientifique contemporaine. A l'heure actuelle, il n'y a plus guère de biologistes qui rejettent la théorie de l'évolution. Il en va de même pour la théorie des feuillets [60]. En ce qui concerne la loi biogénétique, les choses sont différentes : rares aujourd'hui sont les zoologistes qui prennent parti en sa faveur.

C'est que, depuis plusieurs dizaines d'années, la loi biogénétique a mauvaise presse [61]. Suivant un embryologiste de renom, il s'agit d'une théorie "désastreuse" [14]. En fait, c'est moins la loi elle-même qui est critiquable que les dérives qu'elle a suscitées, en particulier en matière de racisme [61]. Par définition, la loi biogénétique définit les rapports entre l'ontogenèse et la phylogenèse. Dans sa version originale, elle stipule que l'ontogenèse récapitule la phylogenèse parce qu'à chaque étape évolutive, de nouveaux mécanismes embryonnaires se sont ajoutés aux mécanismes préexistants par un processus d'addition terminale [62]. Pour Garstang, cette proposition n'est pas défendable. A ses yeux, l'ontogenèse ne récapitule pas la phylogenèse. Elle la crée, puisque la succession des formes adultes est le produit de développements successifs [63]. Nelson propose une version fortement modifiée de la loi biogénétique [64]. Si l'on intègre l'idée de Nelson comme seconde proposition dans un raisonnement en trois points, on arrive à concilier les vues de von Baer, Haeckel et Garstang.

1. Au cours du développement, les caractères généraux se transforment en caractères spécialisés [12]. 2. Les caractères généraux doivent être considérés comme primitifs. Ces caractères étaient présents chez les métazoaires archaïques. Les caractères spécialisés sont apparus plus tardivement [64].

3. Il y a donc au minimum une corrélation entre développement et évolution [62]. En fait, il existe une relation de cause à effet entre ces deux processus [63]. C'est par modifications successives du développement que se sont peu à peu instaurées les différences entre les formes adultes [63]. Par ailleurs, la divergence entre les développements est le fruit d'un processus évolutif. 


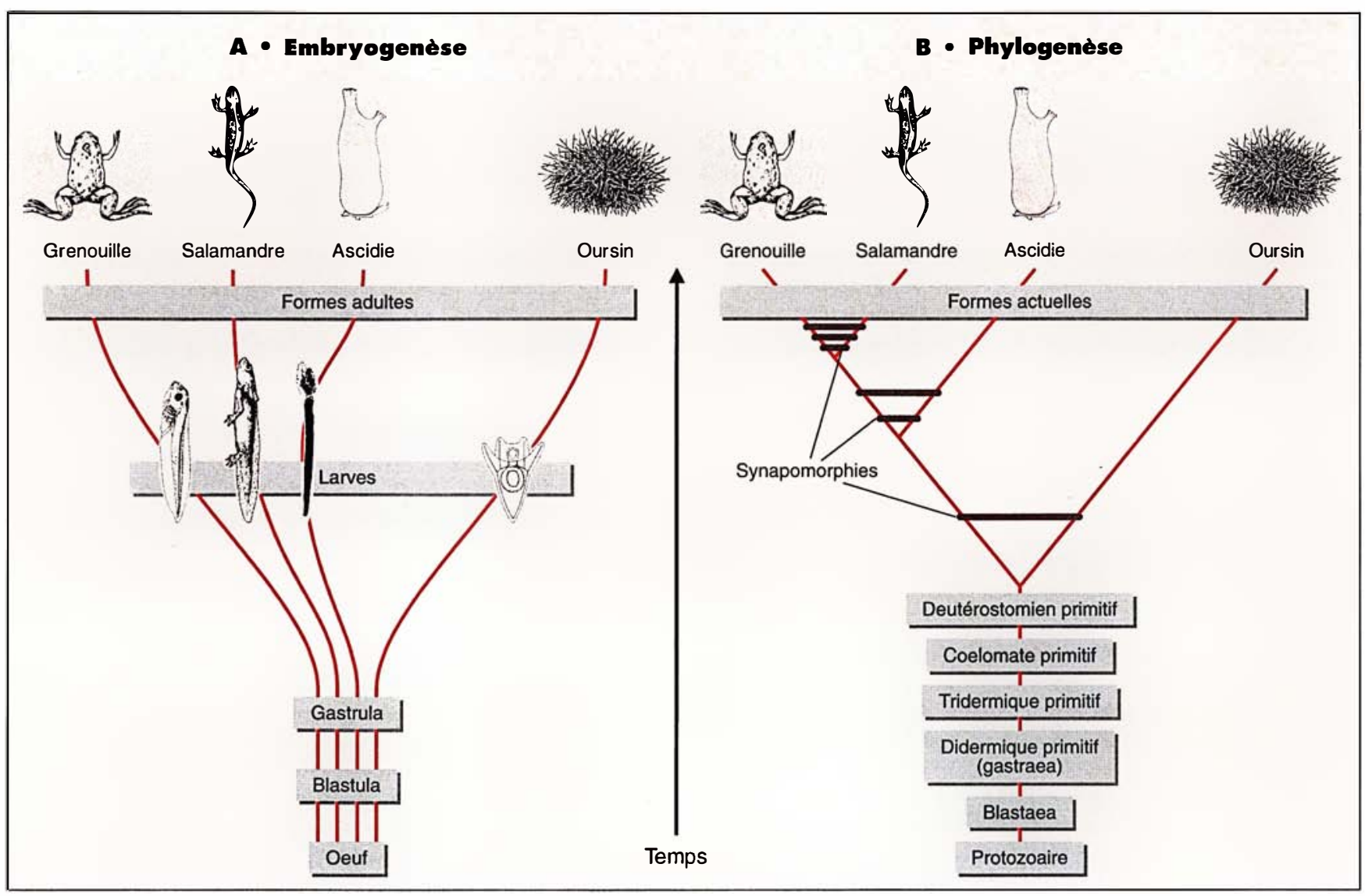

Figure 3. Similitude entre embryogenèse et phylogenèse. Deux arbres similaires mettent en parallèle le développement (A) et l'évolution (B) de quatre deutérostomiens, appartenant à deux phylums: celui des chordés (grenouille, salamandre, ascidie), et celui des échinodermes (oursin). Au cours du développement (A), des différences croissantes apparaissent entre les formes embryonnaires des animaux comparés. Initialement, l'embryogenèse des quatre animaux suit des chemins parallèles: en se divisant, un œuf de forme arrondie se convertit en une blastula, sphère creuse réunissant des cellules semblables par leur structure et leurs fonctions. La blastula évolue ensuite en une gastrula. Le développement commence alors à diverger, si bien que la larve des chordés diffère très nettement de la larve plutéus de l'oursin. Telle qu'on peut la reconstruire, l'évolution (B) fait aussi apparaître une divergence entre les formes adultes des animaux étudiés. Avant la diversification des deutérostomiens, une série de métazoaires primitifs se sont succédé. Parmi ces ancêtres, le plus lointain serait un organisme de type blastaea, ne comportant que deux catégories de cellules: somatiques et germinales. Le plus récent serait un animal tridermique, dont le mésoderme s'organisait en vésicules closes (le cœlome), et dont la bouche ne dérivait pas du blastopore, mais se perçait secondairement. La diversification des deutérostomiens peut se reconstituer grâce à une analyse cladistique des caractères que présentent les formes actuelles. Il s'agit de recenser les synapomorphies, traits dérivés qu'ont en commun les animaux comparés. Moins ces traits sont nombreux, plus les animaux sont censés être éloignés du point de vue phylogénétique. Peu de synapomorphies permettent de rapprocher l'oursin des chordés. Elles concernent la manière dont se déroule le début du développement, et en particulier la gastrulation. II y a davantage de synapomorphies entre l'ascidie et les amphibiens (grenouille et salamandre). Elles se détectent surtout dans les formes juvéniles. Par exemple, les larves d'ascidie et d'amphibien ont une notochorde et une queue. Grenouille et salamandre ont beaucoup de caractères communs. L'une et l'autre possèdent une colonne vertébrale, une mâchoire, quatre pattes, deux oreilles, etc. 


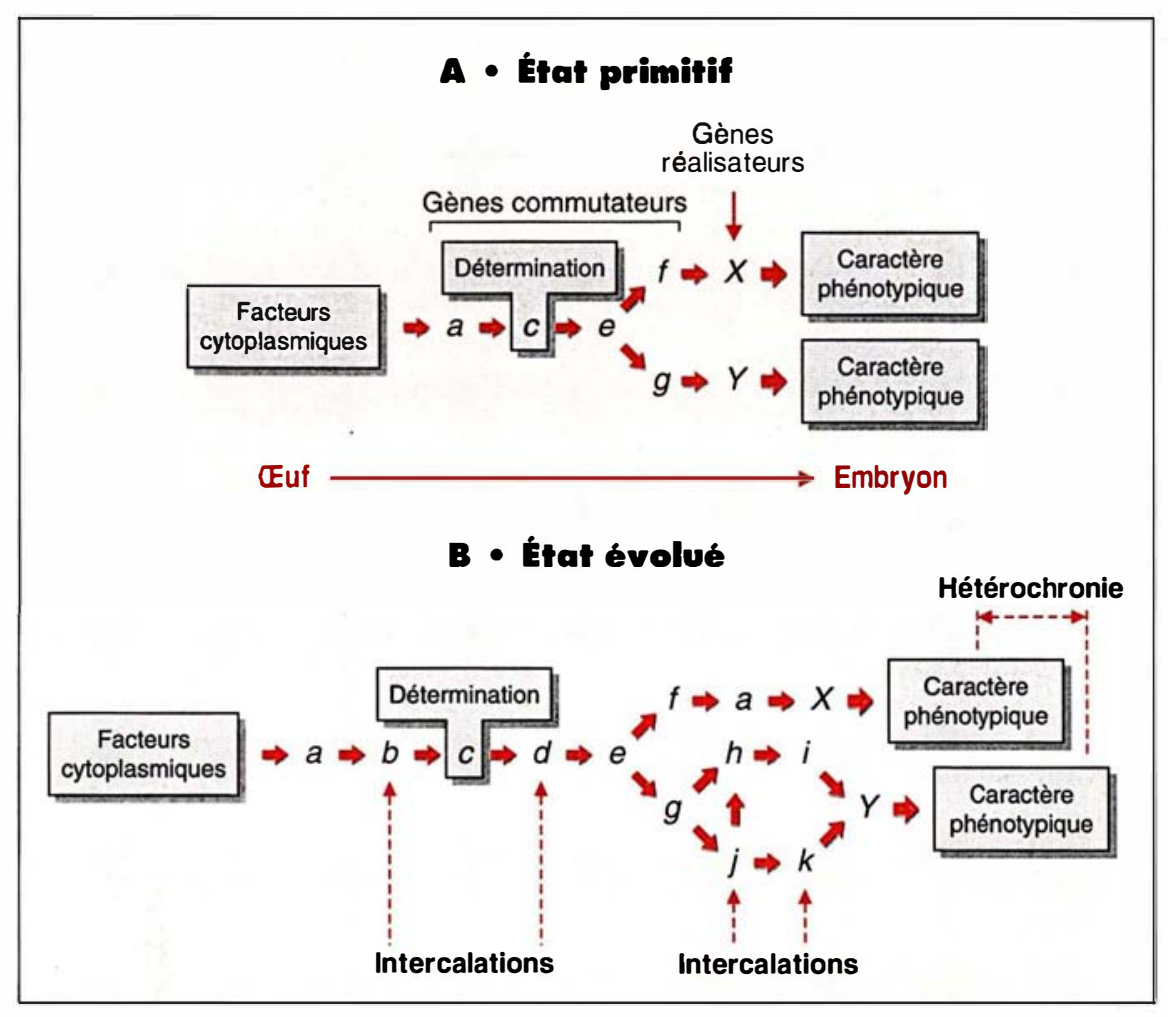

Figure 4. Modification des événements embryonnaires. Nous supposons que depuis l'origine des métazoaires, le développement se déroule selon des modalités comparables. Toutefois, le développement des formes primitives (A) devait faire intervenir moins de gènes que celui des formes évoluées (B). Quelle que soit sa complexité, l'embryogenèse est gouvernée par une cascade de gènes commutateurs et réalisateurs, enclenchés par des facteurs présents dans le cytoplasme de l'œuf [60]. Les commutateurs contrôlent la détermination, tandis que les réalisateurs sont directement responsables de la différenciation cellulaire. Tout changement évolutif implique une modification dans l'embryogenèse, donc dans la cascade de gènes qui dirigent son déroulement. Les gènes anciens évoluent. Certains d'entre eux (tels que a) acquièrent de nouvelles fonctions. $D^{\prime}$ autres disparaissent. De nouveaux gènes (b, d, h, i, j, k) s'ajoutent à la cascade préexistante. II ne peut pas s'agir $d^{\prime}$ une addition terminale, parce que les réalisateurs $(X$ et $Y)$ sont apparemment incapables de modifier l'expression d'autres gènes, et de s'insérer dans une chaîne d'interactions géniques. Par conséquent, la cascade de gènes n'a pu s'enrichir que par intercalation. Ce processus complique les chaînes d'interactions, en les allongeant et en les ramifiant. II crée aussi des hétérochronies, en modifiant la longueur relative des chaînes d'interactions, donc le temps nécessaire pour leur déroulement. L'intercalation peut également amorcer de nouvelles chaînes, qui aboutissent à des réalisateurs supplémentaires. Par souci de simplicité, le nombre de réalisateurs est supposé constant.

\section{Un parallèle entre embryogenèse et phylogenèse}

Il ressort de ce qui précède que les caractères ancestraux apparaissent au début du développement. On ne saurait s'étonner que les études embryologiques et phylogénétiques aient engendré des classifications concordantes. Pour les embryologistes, les groupes les plus proches sont ceux dont l'embryogenèse diverge le plus tardivement. Du point de vue phylogénétique, il s'agit de ceux qui sont apparus le plus récemment au cours de l'évolution.

Nous illustrons notre propos au moyen de deux schémas arborescents qui comparent l'embryogenèse et la phylogenèse de quelques deutérostomiens (figure 3). L'arbre des développements résume les observations faites depuis plus d'un siècle par les embryologistes. C'est une juxtaposition de processus indépendants, qui n'implique aucune filiation entre les animaux examinés. En revanche, l'arbre phylogénétique est censé refléter une telle filiation. Cet arbre se fonde non sur un examen comparé de fossiles qui sont en grande partie manquants - mais sur une analyse cladistique des caractères. La tige présente une succession de formes hypothétiques conduisant à l'ancêtre commun le plus récent des deutérostomiens. Les branches constituent un cladogramme.

Le schéma proposé (figure 3) invoque des phénomènes temporels pour expliquer l'accord entre les deux systèmes de classification proposés par les zoologistes: celui qui s'appuie sur l'étude comparée du développement et celui qui tente de reconstruire la phylogénie. Toutefois, les arbres mis en parallèle diffèrent profondément par les durées et les processus mis en œuvre. Le développement s'accomplit en quelques semaines. Il comporte une succession de mitoses. L'évolution des deutérostomiens se poursuit depuis plusieurs centaines de millions d'années. Elle implique une succession de générations, consistant en une suite ininterrompue de gamétogenèses et d'embryogenèses. 


\section{Gènes commutateurs et récapitulation}

Les progrès récents de l'embryologie incitent à approfondir l'analyse qu'a faite Garstang de la loi biogénétique [63]. Il apparaît que le développement fait intervenir deux catégories de gènes: commutateurs et réalisateurs [60]. Certains commutateurs sont activés par des facteurs localisés dans le cytoplasme de l'œuf (figure 4). Ils activent à leur tour d'autres commutateurs. Les réalisateurs sont situés à l'extrémité des voies d'interactions géniques. Ils spécifient les protéines qui conditionnent la différenciation terminale des cellules. Contrairement aux commutateurs, les réalisateurs semblent inaptes à moduler l'activité d'autres gènes.

Si l'on essaie d'imaginer comment ont évolué les métazoaires, on est amené à penser que leur développement s'est modifié et compliqué principalement par ajout de commutateurs à d'autres commutateurs. Cela conduit à envisager non pas un processus d'addition terminale [62], mais un processus d'intercalation, qui allonge et ramifie les chaînes d'interactions préexistantes, tout en créant des hétérochronies*, c'est-àdire des modifications dans la vitesse relative à laquelle se déroulent les processus du développement (figure 4).

Un exemple d'intercalation peut être trouvé dans l'expression des gènes commutateurs twist et snail chez la drosophile et les vertébrés [67]. Pendant l'embryogenèse de la drosophile, twist et snail sont activés très précocement. Ils jouent un rôle crucial dans la détermination du mésoderme [67]. Dans les embryons du poisson-

\footnotetext{
* Les hétérochronies sont un moteur puissant d'in novation évolutive $[13,61,65,66]$. Les chaînes d'interactions géniques peuvent, soit s'allonger, soit se raccourcir. Un retard apparait si de nouveaux re lais s'interposent (figure 4), ou si divers mécanismes ralentissent l'exécution des ordres émis par les gènes: allongement des séquences introniques, report de la traduction de l'ARN messager, etc. Une accélération se produit si certains relais sont supprimés ou court-circuités. Une hétérochronie ne peut s'instaurer que si les chaines d'interactions restent indépen dantes: toute connexion entre chaines tend $a$ syn-
}

zèbre, du xénope et de la souris, ces gènes interviennent plus tardivement. Ils régissent non pas la détermination du mésoderme, mais sa régionalisation [68-70]. Apparemment, les vertébrés ont interposé des commutateurs supplémentaires en amont de twist et de snail, qui retardent leur action.

L'intercalation tend à modifier la succession ancestrale des processus embryonnaires, donc à faire disparaître toute récapitulation (figure 4). Malgré tout, il semble qu'elle n'a pas complètement bouleversé les chaînes d'interactions géniques dirigeant les étapes initiales du développement. Une certaine conservation se manifeste dans la succession des événements embryonnaires. La formation des feuillets en fournit un exemple [60].

On peut fournir une explication de type néo-darwinien à la conservation des processus initiaux du développement [26]. L'embryogenèse implique une suite de décisions clonales mettant en jeu un nombre croissant de gènes commutateurs, qui s'activent en cascade [60]. Puisque mutations et intercalations (figure 4) sont censées modifier sans discrimination le fonctionnement des gènes, tous les commutateurs sont affectés avec une égale fréquence. Toutefois, il y a peu de chances pour que la sélection naturelle retienne les changements qui touchent les commutateurs agissant au début de l'embryogenèse, parce que ces altérations sont de nature à perturber l'expression de nombreux gènes situés en aval dans la chaîne d'interactions, ce qui conduit à interrompre le développement. En revanche, la survie de l'embryon sera moins souvent compromise par les mutations frappant les commutateurs activés plus tardivement, ainsi que les réalisateurs (figure 4). De telles mutations seront plus fréquemment retenues. En somme, les décisions qui pèsent lourdement sur l'embryogenèse sont susceptibles d'être modifiées plus rarement que les décisions de moindre poids.

\section{Conclusion}

Puisque les caractères ancestraux apparaissent aux premiers stades de l'embryogenèse, il est clair à nos yeux que les progrès futurs de la génétique du développement permettront de préciser les relations taxinomiques entre les grands groupes de métazoaires, et de clarifier l'origine de ces groupes. Nous estimons qu'en élucidant le réseau d'interactions géniques gouvernant l'embryogenèse (figure 4), on obtiendra en matière de taxinomie et de phylogenèse des informations bien plus pertinentes que ne peuvent en apporter les comparaisons de séquences (figure 2). Mais cela nécessitera un effort considérable dans le domaine expérimental et théorique. Il conviendra non seulement d'analyser le réseau de commutation qui dirige l'embryogenèse d'animaux très différents, mais aussi de développer l'analyse mathématique des circuits d'interactions entre gènes [71]

\section{Remerciements}

Les auteurs remercient Richard Christen, Jean Deutsch, Jean-Claude Lacroix, Maryvonne Mével-Ninio, Hervé Philippe, Linda Sperling et Maurice Wegnez pour leurs conseils pendant la rédaction de cet article.

\section{H. Denis}

Professeur à l'université Pierre-et-MarieCurie. Centre de génétique moléculaire Cnrs, avenue de la Terrasse, 91198 Gifsur-Yvette Cedex, France.

\section{A. Collenot}

Professeur retraité de l'université Pierre-etMarie-Curie.

\section{TIRÉS À PART}

\section{H. Denis.}




\section{RÉFÉRENCES}

1. Aristote. Historia animalium. Traduit par J. Tricot. Paris : Vrin, 1987.

2. Aristote. De generatione animalium. Traduit par D'Arcy W. Thompson. Oxford: Oxford University Press, 1912.

3. Hyman LH. The Invertebrates: Protozoa through Ctenophora. New York: McGraw-Hill, 1940 .

4. Linné C. Systema naturae per regna tria naturae, secundum classes, ordines, genera, species cum characteribus, differentiis, synonymis, locis. Editio decima tertia, aucta, reformata. Lyon: Delamollière, 1789 à 1796.

5. Lamarck JBPA. Philosophie zoologique. Paris: Dentu, 1809.

6. Cuvier G. Le règne animal distribué suivant son organisation. Paris: Déterville, 1829.

7. Brusca RC, Brusca GJ. Invertebrates. Sunderland: Sinauer, 1990.

8. Mayr E, Ashlock PD. Principles of systematic zoology. New York: McGraw-Hill, 1991.

9. Margulis L. Symbiosis in cell evolution. New York: Freeman, 1993

10. Grassé PP, Poisson RA, Tuzet O. Zoologie. Tome 1. Invertébrés. Paris: Masson, 1961.

11. Nielsen C. Larval and adult characters in animal phylogeny. Am Zool 1994; 34: 492501.

12. von Baer KE. Ueber Entwicklungsgeschichte der Thiere: Beobachtung und Reflexion. Königsberg: Bornträger, 1828 et 1837.

13. Wolpert $L$. The evolution of development. Biol J Linn Soc 1990; 39: 109-24.

14. Gilbert SF. Developmental biology. Sunderland: Sinauer, 1994.

15. Duboule D. Temporal colinearity and the phylotypic progression: a basis for the stability of a vertebrate Bauplan and the evolution of morphologies through heterochrony. Development Suppl 1994; 135-42.

16. Kenyon C. If birds can fly, why can't we? Homeotic genes and evolution. Cell 1994; $78: 175-80$.

17. Pander C. Beiträge zur Entwickungsgeschichte des Hünchens im Eye. Würtzburg: Brönners, 1817.

18. Remak R. Untersuchungen über die Entwicklung der Wirbelthiere. Berlin: Reimer, 1855.

19. Huxley TH. On the anatomy and affinities of the family of Medusae. Phil Trans $R$ Soc London 1849; 139: 413-34.

20. Ray Lankester E. On the primitive celllayers of the embryo as the basis of genealogical classification of animals, and on the origin of vascular and lymph systems. Ann Mag Nat Hist 1873; 11:321-38.

21. Ray Lankester E. Notes on the embryology and classification of the animal kingdom: comprising a revision of speculations

relative to the origin and significance of the germ-layers. Quart J Micr Sci 1877; 17:399 454 .

22. Kowalevsky A. Weitere Studien über die Entwicklung der einfachen Ascidien. Arch Mikroskop Anat 1871; 7 : 101-30.

23. Grobben K. Die systematische Einteilung des Tierreiches. Verhandl kaiser-könig zool bol Gesell Wien 1908; 58: 491-511.

24. Darwin C. On the origin of species by means of natural selection, or the preservation of favoured races in the struggle for life. Londres: Murray, 1859 .

25. Mayr E. Animal species and evolution. Cambridge: Harvard University Press, 1963.

26. Jefferies RPS. The ancestry of the vertebrates. Londres : British Museum (Natural History), 1986.

27. Gould SJ, Eldredge N. Punctuated equilibrium comes of age. Nature $1993 ; 366: 223-$ 7 .

28. Thomson KS. Spinal discord. Nature $1987 ; 327: 196-7$.

29. Cloud P, Glaessner MF. The Ediacaran period and system: Metazoa inherit the earth. Science 1982; $217: 783-92$

30. Conway Morris S. The fossil record and the early evolution of the Metazoa. Nature $1993 ; 361: 219-25$.

31. Conway Morris S. Burgess shale faunas and the Cambrian explosion. Science 1989 ; $246: 339-46$

32. Bowring SA, Grotzinger IP, Isachsen CE, Knoll AH, Pelechaty SM, Kolosov P. Calibraing rates of early Cambrian evolution. Science $1993 ; 261: 1293-8$.

33. Kerr RA. Evolution's big bang gets even more explosive. Science 1993; 261: 1274-5.

34. Huxley JS. Evolutionary processes and taxonomy with special reference to grades. Uppsala Universitats Arsskrift 1958; 6 : 21-39.

35. Simpson GG. Principles of animal taxono $m y$. New York: Columbia University Press, 1961.

36. Mayr E. Principles of systematic zoology. New York: McGraw-Hill, 1969.

37. Matile L, Tassy P, Goujet D. Biosystema 1. Introduction à la systématique zoologique Paris: Société française de Systématique, 1987.

38. Sneath PHA, Sokal RR. Numerical taxonomy. San Francisco: Freeman, 1973.
39. Tassy P. L'arbre à remonter le temps. Paris: Bourgois, 1991.

40. Hennig W. Grundzüge einer Theorie der phylogenetischen Systematik. Berlin : Deutscher Zentralverlag, 1950 .

41. Gouget D, Matile L, Janvier P, Hugot JP. Biosystema 2. Quelques textes fondamentaux. Glossaire. Paris: Société française de Systématique, 1988 .

42. Zuckerkandl E, Pauling L. Molecular diseases, evolution, and genetic heterogeneity. In : Kasha M, Pullman B, eds. Horizons in biochemistry. Chicago: Academic Press, 1962: 189-225.

43. Zuckerkandl E, Pauling L. Evolutionary divergence and convergence in proteins. In : Bryson V, Vogel HJ, eds. Evolving genes and proteins. New York: Academic Press, 1965: 97-165.

44. Jukes TH. Transitions, transversions, and the molecular evolutionary clock. $J$ Mol Evol $1987 ; 26: 87-98$

45. Field KG, Olsen GJ, Lane DJ, Giovannoni SJ, Ghiselin MT, Raff EC, Pace NR, Raff RA. Molecular phylogeny of the animal kingdom. Science 1988; 239: 748-53.

46. Gouy M, Li WH. Molecular phylogeny of the kingdoms Animalia, Plantae, and Fungi. Mol Biol Evol 1989; 6 : 109-22.

47. Lake IA. Origin of the Metazoa. Proc Natl Acad Sci USA 1990; 87: 763-6.

48. Christen R, Ratto A, Baroin A, Perasso R, Grell $\mathrm{KG}$, Adoutte A. An analysis of the origin of metazoans, using comparisons of partial sequences of the $28 \mathrm{~S}$ RNA, reveals an early emergence of triploblasts. $E M B O J$ 1991 ; $10: 499-503$.

49. Turbeville JM, Field KG, Raff RA. Phylogenetic position of phylum Nemertini, inferred from $18 \mathrm{~S}$ rRNA sequences : molecular data as a test of morphological character homology. Mol Biol Evol 1992; 9: $235-49$

50. Wainright PO, Hinkle G, Sogin ML, Stickel SK. Monophyletic origins of the Metazoa : an evolutionary link with fungi. Science $1993 ; 260: 340-2$

51. Baldauf SL, Palmer JD. Animals and fungi are each other's closest relatives : congruent evidence from multiple proteins. Proc Natl Acad Sci USA 1993; 90: 11558-62.

52. Adoutte A, Philippe $H$. The major lines of metazoan evolution: summary of traditional evidence and lessons from ribosomal RNA sequence analysis. In: Pichon $\mathrm{Y}$, ed. Comparative molecular neurobiology. Bâle: Birckhäuser, 1993: 1-30.

53. Telford MJ, Holland PWH. The phylogenetic affinities of the Chaetognaths: a molecular analysis. Mol Biol Evol 1993; 10:660 76. 
54. Wada H, Satoh H. Details of the evolutionary history from invertebrates to vertebrates, as deduced from the sequences of 18S RNA. Proc Natl Acad Sci USA 1994; 91 . $1801-4$.

55. Philippe $\mathbf{H}$, Chenuil A, Adoutte A. Can the Cambrian explosion be inferred through molecular phylogeny? Development Suppl 1994 ; 15-25.

56. Winnepenninckx B, Backeljau T, De Wachter R. Phylogeny of protostome worms derived from $18 \mathrm{~S}$ rRNA sequences. Mol Biol Evol $1995 ; 12$ : 641-9.

57. Fitch WM, Margoliash E. Construction of phylogenetic trees. Science 1967; 155:279 84 .

58. Darlu P, Tassy P. Reconstitution phylogéné tique. Concepts et méthodes. Paris: Masson, 1993.

59. Hyman LH. The Invertebrates: smaller coe lomate groups. New York: McGraw-Hill, 1959.

60. Denis H, Collenot A. La théorie des feuillets: implications embryologiques et évolutives. médecine/sciences 1995; 11: 1581-93.

61. Gould SJ. Ontogeny and phylogeny. Cambridge: Harvard University Press, 1977.

62. Haeckel E. Die Gastraea-Theorie, die phylogenetische Classification des Thierreichs und die Homologie der Keimblätter. Jenaische Z Naturwiss $1874 ;$; : 1-55.
63. Garstang W. The theory of recapitulation : a critical re-statement of the biogenetic law. J Linn Soc Zool 1922; 35: 81-101.

64. Nelson G. Ontogeny, phylogeny, paleontology, and the biogenetic law. Syst Zool $1978 ; 27: 324-45$.

65. Raff RA, Kaufman TC. Embryos, genes, and evolution. New York: Macmillan, 1983.

66. McKinney ML, McNamara KJ. Heterochrony. The evolution of ontogeny. New York: Plenum Press, 1991.

67. Reuter R, Leptin M. Interacting functions of snail, twist and huckebein during the early development of germ layers in Drosophila. Development 1994; 120: 1137-50.

68. Hammerschmidt M, Nüsslein-Volhard C The expression of a zebrafish gene homologous to Drosophila snail suggests a conserved function in invertebrate and vertebrate gastrulation. Development 1993; 119: 1107-18.

69. Hopwood ND, Pluck A, Gurdon JB. A Xenopus mRNA related to Drosophila twist is expressed in response to induction in the mesoderm and the neural crest. Cell 1989 59: 893-903.

70. Chen ZF, Behringer RR. twist is required in head mesenchyme for cranial neural tube morphogenesis. Genes Dev 1995; 9: 686-99.

71. Thomas R. Regulatory networks seen as asynchronous automata : a logical description. J Theor Biol 1991; 153: 1-23.

\section{Summary}

Taxonomy and embryology: an ancient and fruitful connection

The current system of animal classification relies on the number and structure of the germ layers built by early embryos. This system dates back to the beginning of the XXth century. In spite of the increasing influence exerted by phylogenetic studies on taxonomy, there has been no fundamental modification of the development-based system of classification. This is not surprising. A certain parallelism exists between development and evolution. When related animals are compared, more similarities can be detected between their embryonic forms than between their adult ones. Like animals have late diverging developments; unlike animals have early diverging developments. A similar trend can be discerned in evolution. Closely related groups have a recent origin; distantly related groups have a more ancient origin. Therefore, a development-based taxonomy is likely to reflect phylogeny. 